\title{
Using affective knowledge to generate and validate a set of emotion-related, action words
}

Emma Portch, Jelena Havelka, Charity Brown, Roger Giner-Sorolla

Emotion concepts are built through situated experience. Abstract word meaning is grounded in this affective knowledge, giving words the potential to evoke emotional feelings and reactions (e.g. Vigliocco et al., 2009). In the present work we explore whether words differ in the extent to which they evoke 'specific' emotional knowledge. Using a categorical approach, in which an affective 'context' is created, it is possible to assess whether words proportionally activate knowledge relevant to different emotional states (e.g. 'sadness', 'anger', Stevenson, Mikels \& James, 2007a). We argue that this method may be particularly effective when assessing the emotional meaning of action words (e.g. Schacht \& Sommer, 2009). In study 1 we use a constrained feature generation task to derive a set of action words that participants associated with six, basic emotional states (see full list in Appendix A). Generation frequencies were taken to indicate the likelihood that the word would evoke emotional knowledge relevant to the state to which it had been paired. In study 2 a rating task was used to assess the strength of association between the six most frequently generated, or 'typical', action words and corresponding emotion labels. Participants were presented with a series of sentences, in which action words (typical and atypical) and labels were paired e.g. "If you are feeling 'sad' how likely would you be to act in the following way?" ... 'cry'. Findings suggest that typical associations were robust.

Participants always gave higher ratings to typical vs. atypical action word and label pairings, even when (a) rating direction was manipulated (the label or verb appeared first in the sentence), and (b) the typical behaviours were to be performed by the rater themselves, or others. Our findings suggest that emotion-related action words vary in the extent to which they evoke knowledge relevant for different emotional states. When measuring affective grounding, it may then be appropriate to use categorical ratings in conjunction with unimodal measures, which assess the 'magnitude' to which words evoke feelings (e.g. Newcombe et al., 2012). Towards this aim we provide a set of emotionrelated action words, accompanied by generation frequency and rating data, which show how strongly each word evokes knowledge relevant to basic emotional states. 

Emma Portch, School of Psychology, University of Leeds, Leeds, UK. Jelena Havelka, School of Psychology, University of Leeds, Leeds, UK Charity Brown, School of Psychology, University of Leeds, Leeds, UK

Roger Giner-Sorolla, School of Psychology, Keynes College, University of Kent, Canterbury, 8

Corresponding Author: Emma Portch. A: School of Psychology, Lifton Place, University of Leeds, Leeds, LS2 9JT. T: +44 (0)113343 2275, E: e.s.portch@leeds.ac.uk 


\section{Introduction}

25 Emotion words are not just 'words'. Recent theories of semantic representation suggest that

26 abstract words (including emotional words) are predominantly understood due to their grounding

27 in situated experience (e.g. Vigliocco et al., 2009; Wilson-Mendenhall et al., 2011; Vinson,

28 Ponari \& Vigliocco, 2014). Words derive meaning because they are bound to the emotional

29 experiences that they refer to; words have the power to re-activate or evoke these internal

30 feelings or states (Wilson-Mendenhall et al., 2011). For example, the word 'Justice' is

31 understood because it easily evokes certain emotional connotations, such as feelings commonly

32 associated with receiving a jury verdict (e.g. joy, frustration, dismay; example taken from

33 Newcombe et al., 2012). This parallels the proposed situated conceptualisation of concrete words

34 (e.g. 'pen'), which predominantly find grounding in their sensorimotor bindings (e.g. what a

35 'pen' looks like and how we interact with this object in our environment e.g. Barsalou, 1999;

36 Barsalou et al., 2008).

37 Several findings support the proposed distinction in the types of knowledge that ground abstract

38 and concrete words (e.g. Vigliocco et al., 2009; Wiemer-Hastings \& Xu, 2005; Newcombe et al.,

39 2012). Using a feature generation task, Wiemer-Hastings and Xu (2005) showed that participants

40 generated a significantly higher number of 'experience' and feeling-related properties when

41 defining abstract concepts in comparison to concrete concepts, which elicited a larger proportion

42 of 'entity' and 'situational' properties. Findings from lexical decision tasks also show that

43 dimensional, affective ratings (valence and arousal, e.g. Lang, 1980) are better predictors of

44 abstract than concrete word recognition (Kousta, Vinson \& Vigliocco, 2009; Kousta et al.,

45 2011). In contrast to the classic finding, abstract words were processed faster than concrete

46 words when other types of experiential property were controlled (e.g. imageability ratings for 
47 each word). Importantly, Vinson, Ponari \& Vigliocco (2014) also show that valence is similarly 48 predictive of lexical decision latencies when participants responded both to emotion labels (e.g.

49 'Sad') and emotion-related words (e.g. 'Death'), showing that affective knowledge provides an

50 important binding for words possessing both strong and weak associations to emotional concepts

51 (see also Altarriba \& Basnight-Brown, 2010).

52 These findings suggest that dimensional ratings (valence and arousal e.g. Lang, 1980) effectively 53 quantify the affective knowledge that ground abstract words (e.g. Kousta, Vinson \& Vigliocco, 54 2009; Kousta et al., 2011; Vinson, Ponari \& Vigliocco, 2014). They build on a large body of 55 previous work, showing that dimensional ratings well predict differences in neutral vs. emotional word processing, using the lexical decision paradigm (e.g. Estes \& Adelman, 2008; Larsen et al., 2008; Kousta, Vinson \& Vigliocco, 2009), even when very large sets of words are sampled (e.g. Vinson, Ponari \& Vigliocco, 2014; Kuperman, 2014; Kuperman et al., 2014). However, some researchers explore the utility of new variables. In particular, Newcombe et al., (2012) developed a semantic richness measure, called 'emotional experience'. This measure is similar to bodyobject interaction (Siakaluk et al., 2008) and imageability scales (Schock, Cortese \& Khanna, 2012), which assess the extent to which words represent and elicit the experiential properties of their referents (in the latter case, sensory and motor properties). As such, the emotional experience variable captures the ease with which a word evokes affective knowledge. Newcombe et al., (2012) collected participant-generated emotional experience ratings for a large set of nouns, predictably finding that emotional experience ratings were higher for abstract than concrete words (Moffat et al., 2015; Vigliocco et al., 2009). 
70 Moffat et al., 2015). For example, participants were significantly slower to process abstract

71 words rated high (vs. low) in emotional experience in a Stroop task, suggesting a larger degree

72 of interference when processing words with strong links to a potentially large pool of affective

73 information. Additionally, when participants engaged in a semantic categorisation task, in which

74 they categorised either 'abstract' or 'concrete' words in a continuous stream, high emotional

75 experience ratings were related to facilitative effects in the abstract categorisation task, and

76 smaller inhibitory effects in the concrete categorisation task (Newcombe et al., 2012; Moffat et

77 al., 2015). Importantly, emotional experience ratings continued to predict all three effects, even

78 when valence and arousal ratings were entered as predictors (Newcombe et al., 2012; Siakaluk,

79 Knol \& Pexman, 2014; Moffat et al., 2015). Taken together, these findings show that emotional

80 experience ratings provide a valid way to quantify affective, experiential knowledge (e.g.

81 Newcombe et al., 2012).

82 Categorical ratings present a related way to assess affective grounding. Arguably, emotional

83 experience ratings provide an 'undifferentiated' quantification, suggestive of the ease with which

84 a word evokes knowledge relevant to a range of emotional states e.g. the word 'funeral' may

85 strongly evoke feelings relevant to different emotions, like 'sadness', 'anguish' and 'fear'

86 (Newcombe et al., 2012). Put another way, emotional experience ratings quantify the magnitude

87 to which a word makes you 'feel' (Siakaluk, Knol \& Pexman, 2014). However, we might be

88 explicitly interested in the likelihood that the word 'funeral' evokes feelings of 'sadness', in

89 proportion to feelings and knowledge related to other possible states, like 'fear' and 'anguish'.

90 By posing the emotional label of 'sadness', a constrained 'affective context' is created, under

91 which participants' judge the specific relationship between the emotional concept of 'sadness',

92 and the word 'funeral'. This conceptualisation brings a greater degree of specificity to the notion 
93 that words evoke feelings. In this sense, categorical ratings are similar to context availability

94 measures, which assess the likelihood that words evoke contexts (or, emotional states), in which

95 their referents appear (e.g. Schwanenflugel \& Shoben, 1983; Wiemer-Hastings \& Xu, 2005;

96 Moffat et al., 2015). Additionally, categorical ratings support Pecher, Boot and van Dantzig's

97 (2011) view of how abstract concepts are grounded. They emphasise that abstract words likely

98 reactivate very specific contexts or situations that we have experienced, rather than being

99 generally evocative; just as concrete words reactivate specific sensory and motor representations,

100 in the same neural areas that initially process sensorimotor information (e.g. Wilson-Mendenhall

101 et al., 2011).

102 Several researchers already provide categorical ratings for emotional words (e.g. Stevenson,

103 Mikels \& James, 2007a; Briesemeister, Kuchinke \& Jacobs., 2011a). In Stevenson, Mikels \&

104 James' (2007a) study participants rated each word in the ANEW database (Bradley \& Lang,

105 1999), based on extent of association with the basic states of 'happiness', 'sadness', 'anger',

106 'fear' and 'disgust' (e.g. Ekman, 1992). Here discrete emotional states, denoted by a label, create

107 a constrained 'affective context' and participant ratings indicate the likelihood to which each

108 ANEW word proportionally evokes knowledge relevant to those emotional states. Stevenson,

109 Mikels and James (2007a) were particularly interested in whether words could be 'discretely'

110 related to a particular emotion label. Given that words likely evoke experiential knowledge

111 relevant to a number of emotional states (e.g. Siakaluk, Knol \& Pexman, 2014), we reframe

112 Stevenson, Mikels and James (2007a) terminology to talk about 'disproportional' relationships

113 (e.g. how strongly does a word evoke knowledge relevant to one basic emotion state, in

114 comparison to others?) ${ }^{1}$. Stevenson, Mikels and James (2007b) assumed that a disproportional

\footnotetext{
${ }^{1}$ We acknowledge that the word 'categorical' has strong, dichotomous connotations; something is either part of a category, or it is not. Although we argue for a proportional, rather than a categorical, interpretation we continue to use the word 'categorical' to describe our approach due to its strong relationship with other work that has used this
} 
115 association was present when the rating given for the word/label pair was one standard deviation

116 higher than ratings given to that word when paired with all other emotion labels. Using this

117 method $44.54 \%$ of the 1,034 words tested were disproportionally related to one or two discrete

118 emotion labels. Briesemeister, Kuchinke \& Jacobs, (2011a) produced similar findings when

119 using this rating method with German nouns included in the Berlin Affective Word List (Võ,

120 Jacobs \& Conrad, 2006; Võ et al., 2009). When Stevenson, Mikels and James (2007a) criterion

121 was applied, $25.18 \%$ of the words within DENN-BAWL could be disproportionately associated

122 with a particular emotion label.

123 Importantly, subsequent work shows that categorical ratings for both English and German words

124 predicted lexical decision latencies (e.g. Briesemeister Kuchinke \& Jacobs, 2011a; 2011b; 2014).

125 In particular, words disproportionately related to the discrete state of 'happiness' were processed

126 faster than neutral words and words disproportionately associated with negative discrete

127 categories, like 'disgust', 'fear' (Briesemeister, Kuchinke \& Jacobs, 2011a) and 'anger'

128 (Briesemeister, Kuchinke \& Jacobs, 2011b). Briesemeister, Kuchinke \& Jacobs, (2014) and

129 Briesemeister et al., (2014) both provide evidence to suggest that behavioural facilitation was not

130 simply driven by the positive valence of these words. Temporally dissociable ERP components

131 (Briesemeister, Kuchinke \& Jacobs, 2014) and topographically distinct brain activity

132 (Briesemeister et al., 2014) were found when participants processed words that differed in

133 'happiness' association (high vs. low), but were matched on valence and arousal.

134 A recent study by Westbury et al., (2014) provides further support for the categorical approach.

135 Rather than using participant ratings, Westbury et al., (2014) mapped the semantic distance

136 between emotion labels and words, based on how frequently they co-occurred in close proximity

terminology e.g. Stevenson, Mikels and James (2007a); Briesemeister, Kuchinke \& Jacobs, (2011a). 
137 within a large corpus of text (HiDeX; e.g. Shaoul \& Westbury, 2010). According to Vigliocco et

138 al's., (2009) theory of semantic representation, linguistic co-occurrence supplements experiential

139 grounding of abstract words, pairing affective components whose referents we may not have

140 directly experienced (e.g. knowing that funerals evoke feelings of sadness arguably relies on

141 having attended a funeral; see also Barsalou et al., 2008). First, Westbury et al., (2014) found

142 that the dimensional ratings for a large subset of words (Warriner, Kuperman \& Brysbaert, 2013)

143 could be partially predicted by the quantified linguistic co-occurrence between those words and

144 an accepted set of emotion labels. Second, they found that these co-occurrence values could be

145 used to predict lexical decision latencies for those words (taken from the English Lexicon

146 Project, Balota et al., 2007). In some cases, co-occurrence values were better predictors of

147 latency than valence and arousal ratings, particularly when considering co-occurrence with the

148 emotion labels 'pleasant' and 'unpleasant'.

149 These investigations suggest that categorical ratings, or measures which quantify the

150 proportional association between emotion labels and words, are useful for characterising the way

151 abstract words are processed. Although some findings may be interpreted in a way to suggest

152 that categorical ratings capture different aspects of emotional word processing than standard

153 dimensional variables (e.g. Stevenson, Mikels \& James, 2007a; Briesemeister, Kuchinke \&

154 Jacobs, 2011b, 2014; Briesemeister, Kuchinke \& Jacobs, 2014; Westbury et al., 2014) it is

155 beyond the scope of the present work to assess the relationship between, or relative merits of the

156 two approaches (see also Newcombe et al., 2012). Importantly though, we do suggest that

157 categorical ratings may be particularly useful for quantifying the affective grounding of emotion

158 verbs, or action-related words. Here we single out words which describe behaviours related to

159 particular emotional states, without naming the emotion itself (Pavlenko, 2008). It is not yet 
160 possible to test this proposal as studies using the DENN-BAWL focus exclusively on emotional

161 nouns (Briesemeister, Kuchinke \& Jacobs, 2011a; Briesemeister, Kuckinke \& Jacobs 2014).

162 Further it is unclear whether nouns, adjectives and verbs were equally sampled when

163 Briesemeister, Kuchinke and Jacobs (2011b) selected words from Stevenson, Mikels and James'

164 (2007a) categorisation of the ANEW, or when Westbury et al., (2014) sampled from HiDeX

165 (e.g. Shaoul \& Westbury, 2010).

166 We argue that emotion-related action words hold a special kind of relationship with experiential

167 knowledge. On the one hand these words may be classified as 'concrete'. According to

168 Vigliocco et al's., (2009) framework then, verb meaning should be predominantly situated in

169 sensorimotor knowledge and understood by reactivation in visual and motor areas (e.g.

170 Pulvermüller, 1999; Hauk, Johnsrude \& Pulvermüller, 2004). In support, various researchers

171 show that processing of words directly related to emotional expressions and behaviours e.g.

172 'smile', activate face and body-specific regions for performing that action (e.g. Niedenthal et al.,

173 2009; Moseley et al., 2012) and improve understanding of these expressions, when shown by

174 actors (e.g. Foroni \& Semin, 2009; Halberstadt et al., 2009). On the other hand, verbs that refer

175 to emotional actions are still 'emotional' in nature (Altarriba \& Basnight-Brown, 2010; Vinson,

176 Ponari \& Vigliocco, 2014). Wilson-Mendenhall et al., (2011) emphasise that 'affective',

177 experiential knowledge is necessarily multi-faceted, as it is built within the context of situated

178 activity, and thus include various actions and bodily sensations. Therefore, words referring to

179 emotional actions are likely grounded in both sensorimotor and affective, experiential

180 knowledge.

181 Due to their dual-experiential-representation, it may be important to make an 'affective context'

182 salient when attempting to measure the affective grounding of words that refer to emotional 
183 actions. This additional step is less necessary when presenting more 'abstract' emotional words,

184 such as nouns, which have weaker sensorimotor grounding (e.g. Vigliocco et al., 2009). Nouns

185 like 'cancer', 'death' and 'funeral' are likely to spontaneously evoke unambiguous, negative

186 affective knowledge, even when presented in isolation (e.g. Pavlenko, 2008; Vinson, Ponari \&

187 Vigliocco, 2014), which makes it highly appropriate to use standard dimensional or emotional

188 experience ratings to capture their emotional meaning (e.g. Newcombe et al., 2012). However,

189 when the verb 'jump' is presented alone several alternative, but equally acceptable emotional

190 interpretations are available, as the word has both positive and negative connotations. For

191 example, while someone might 'jump for joy', they may also jump in reaction to a surprising or

192 fearful stimulus.

193 Physiological evidence supports the notion that it is comparatively difficult to extract emotional

194 meaning from isolated verbs. Comparing across paradigms, the event-related potentials

195 commonly associated with early and late semantic processing of single emotional words (e.g.

196 Herbert et al., 2006) are commonly evidenced at a later onset for emotional verbs (Schacht \&

197 Sommer, 2009; Palazova et al., 2011) than for emotional nouns (e.g. Kanske \& Kotz, 2007;

198 Kissler et al., 2007) or adjectives (Herbert et al., 2006; Herbert, Junghöfer \& Kissler, 2008).

199 With reference to the previous example, emotional meaning is easier to interpret when more

200 information is available to provide an 'affective context' e.g. if we know that the actor jumped

201 because 'the car crashed into the nearby lamppost.' In this case, the 'jump(ing)' behaviour is

202 likely related to a negative emotional state, most likely to be 'fear'. In support, Schacht and

203 Sommer (2009) reported Early Posterior Negative (EPN) and Late Positive Complex (LPC)

204 onsets comparable to those for emotional nouns and adjectives when a clear, 'affective context'

205 was applied. Here participants responded to a verb preceded by a noun (e.g. 'lover-kiss'). 
206 Schacht and Sommer (2009) argue that the preceding noun improved participants' ability to

207 extract the intended, emotional meaning from test verbs during a lexical decision task. Applying

208 a similar manipulation, Palazova, Sommer and Schacht, (2013) found comparable EPN onsets

209 when emotional verbs referred to more concrete, context-invariant behaviours, which had clear

210 affective connotations (e.g. to dance vs. to hope).

211 The present work aims to explore whether a categorical approach can be used to examine the

212 affective, experiential knowledge that partially grounds action word meaning. Importantly, in the

213 first study we pose basic emotion labels (e.g. 'Sad') to create a constrained 'affective context'.

214 Participants will self-generate emotional action words that they commonly associate with each

215 emotional state. Generation frequencies, per action word, will be indicative of the likelihood that

216 the word evokes affective, experiential knowledge relevant to paired emotion labels. In the

217 second study a rating task will be conducted to validate use of generation frequencies as a

218 measure of associative strength. Verbs are paired with the emotional labels to which they have

219 been most disproportionately generated, and rated according to the strength of that association.

220 This work provides relevant research communities (e.g. researchers interested in both emotion

221 and language processing) with a database of emotion action words. Accompanying generation

222 frequency (study 1) and rating data (study 2) are suggestive of the extent to which these words

223 evoke affective knowledge related to a set of basic emotional states.

224

225 Study 1- Identifying action words that proportionally evoke affective knowledge

226 In study 1 we use emotion labels to provide a constrained, 'affective context'. Following

227 Stevenson, Mikels and James., (2007a) and Briesemeister, Kuchinke and Jacobs, (2011a), we

228 present the universal, basic emotion labels used by Ekman (1992; 'happy', 'sad', fear', 'anger', 
229 'disgust' and 'surprise'). We reason that these states represent commonly experienced emotions

230 which will be fluently associated with behavioural referents.

231 Rather than use a rating task, we conduct a highly constrained semantic feature-generation task.

232 Participants are instructed to self-generate multiple single-word actions that they commonly

233 associated with experiencing each of these discrete emotional states (see McRae et al., 2005;

234 Vinson \& Vigliocco, 2008 and Buchanan et al., 2013 for broader examples of semantic feature

235 generation $^{2}$ ). Explicit instructions were important as action words have rarely been produced

236 when emotion labels are posed as 'concepts' in feature generation tasks (e.g.; Hutchison et al.,

237 2010; Buchanan et al., 2013). By encouraging participants to engage separately with each

238 emotion label we also hoped to widen the stimulus set, as rating methods often produce a

239 'happiness asymmetry' (many words are strongly associated with 'happiness', but far fewer

240 words are associated with discrete, negative states e.g. Stevenson, Mikels \& James, 2007a;

241 Briesemeister, Kuchinke \& Jacobs, 2011a).

242 Overall, we measure the likelihood that an action word evokes discrete affective knowledge

243 based on the frequency of participants who endorse the pair (e.g. McRae et al., 2005). However,

244 we acknowledge that the ability to infer proportional association also relies on the number of

245 additionat emotionat states to which the action word is generated.

${ }^{2}$ We acknowledge that similar methods have been used to elicit related stimuli, such as action-readiness and tendency items (Smith \& Ellsworth, 1985; Frijda, 1986; Frijda, Kuipers \& Ter Schure, 1989). However, these items usually refer to a general anticipatory state that the individual enters after appraising an emotionally salient event (Frijda, 1986). Although important components of affective knowledge, these items are generally dissociable from the concrete, overt behaviours derived in the present study, which may be viewed as the eventual behavioural consequence of experiencing such states. 


\section{Method}

247 Ethics

248 This research is subject to ethical guidelines set out by the British Psychological Society (1993)

249 and was approved by the School of Psychology's ethics committee, at the University of Leeds

250 (reference number: 13-0032, date of approval: 24/02/2013).

\section{Participants}

252 Twenty-five participants (17 female, 8 male) generated action words. Participants had a mean

253 age of $27.24(\mathrm{SD}=7.63)$ and all reported themselves to be native English speakers (7 participants

254 spoke a second language, though did not consider themselves fluent). An opportunity

255 recruitment method was used. Participants responded to links posted on research recruitment

256 websites and completed the study online (e.g.

257 http://www.psych.hanover.edu/research/exponnet.html; http://www.onlinepsychresearch.co.uk;

258 http://www.in-mind.org/content/online-research; http://www.reddit.com/r/SampleSize).

259 Procedure

260 All materials, including informed consent items, were presented using the Survey Monkey

261 platform (http:/www.surveymonkey.com, Survey Monkey Inc. Palo Alto, California, USA).

262 Participants ticked a series of boxes to confirm that they understood task instructions and gave

263 their informed consent to take part. Participants were then asked to carefully read the definition

264 of an emotion-related action word, below (taken from Pavlenko, 2008). Definitions were edited

265 to include relevant examples. 
'Emotion-related' words are used to describe behaviours related to a particular emotional state,

without naming the actual emotion. For example, the word 'cry' might describe the behaviour of

268

someone feeling sad while the word 'smile' may describe the behaviour of somebody who is

happy.'

Participants were directed to six basic emotion labels, listed below the definition ('sad', 'happy', 'anger', 'disgust', 'surprise' and 'fear', Ekman, 1992). They were asked to generate as many emotional action words as they could which were related to each basic label. Separate boxes were provided for participants to type their examples. Participants were instructed to provide single-word answers and to avoid label synonyms or adverbs (e.g. 'sadness', 'sadly'). They were also discouraged from using the internet to generate responses. Participants were asked to work on the basic labels sequentially and labels were presented in a randomised order across participants. There was no time limit imposed on word generation.

\section{Results: Data modifications and modal exemplars}

In total, participants generated 362 unique words, across the six labels. On average, participants each generated 27.32 words during the task $(S D=15.18)$. We parsed the data in various ways to determine an acceptable set of action words, which were 'modally' associated with one or more 
word with the same or similar meaning, for example, 'sharp intake or breath' was replaced with 'gasp'. Third, merging techniques were used either when participants provided grammatical

291 derivatives or plurals of the same word (e.g. 'ran', 'run', 'runs', 'running', 'ran away') or

292 generated synonyms for action words that had already been provided by themselves or others

293 (e.g. 'scream' and 'shriek'). In the former case, plurals were changed to their singular form and

294 grammatical derivatives were merged and represented by the simplest version, provided their

295 meaning did not change (e.g. 'run').

296 The second type of merging (non-derivative words) was wholly motivated by our need to

297 develop stimuli for study 2 . Here we required only six action words, each of which held the most

298 disproportional association with one of the six emotion labels, respectively. Therefore it was

299 important to ensure that words with the same/very similar meanings were grouped together, and

300 their frequencies summed, to aid assessment of how strongly those related behaviours evoked

301 discrete, affective knowledge ${ }^{3}$. Strict criteria were imposed for this form of merging. Action

302 words were only classed as synonymous if there was evidence of forward and backward

303 association e.g. when 'laugh' was entered into the thesaurus 'giggle' was given as a synonym,

304 and when 'giggle' was entered into the thesaurus, 'laugh' was given as a synonym. We were

305 mindful that some action words could have multiple meanings when presented in isolation (e.g.

306 Schacht \& Sommer, 2009). For example, the action word 'jump' could mean 'to leap, spring or

307 skip', 'to recoil' or 'to avoid' (definitions taken from http://www.thesaurus.com). In these cases

308 the participants' intended meaning was discerned by considering the emotion label to which the

\footnotetext{
${ }^{3}$ Although this type of merging helped to identify the top-six modal action words, for use in study 2, it necessarily inflated the apparent frequency-based strength of association between those core action words and corresponding emotion labels. Readers are encouraged to consult Appendix A, in which all modal exemplars are listed alongside unmerged generation frequencies, which provide a clearer estimation of the strength with which individual action works evoke affective knowledge relevant to different emotion states. From appendix A, researchers may select stimuli based on unmerged exemplars, or apply their own criteria to identify and merge synonymous exemplars.
} 
309 word had most frequently been generated. As the word 'jump' was frequently endorsed for the

310 labels 'surprise' and 'fear' it went unmerged with 'skip', which although a synonym, was only

311 given in response to the label 'happy'. Here we considered that the two words likely had a

312 different intended meaning, each congruent with the core emotion concept to which they had

313 been modally generated (see Buchanan et al., 2013 for similar consideration of 'cue' word when

314 merging 'target' words).

315 Where merging occurred, frequencies for both/all action words were added together. For non-

316 derivative synonyms the dominant response was retained, based on existing frequencies (i.e. the

317 action word given by the highest number of participants).This exemplar became the 'core' action

318 word and non-dominant responses were subsumed and became 'subsidiary' action words. For

319 example, in response to the label 'sad', 'cry' became a core action word and the synonyms

320 'weep' and 'sob' became subsidiaries'. The number of participants who generated the action

321 words 'cry', 'weep' and 'sob' were added together to provide a frequency total for the core

322 action word ('cry'). Note that frequencies could exceed 25 if participants had provided both core

323 and subsidiary action words in response to the same emotion label.

324 Following these steps our set still contained a large number of 'idiosyncratic' responses,

325 generated by only one participant in response to a particular label (124 words, $56.88 \%$ of

\footnotetext{
${ }^{4}$ It was particularly difficult to make merging decisions about the exemplar 'cry'. As this exemplar was given in response to the 'sad', 'anger', 'fear', 'happy' and 'surprise' categories, consideration of cue word could result in two (or more) definitions being accepted. To illustrate, when generated in response to 'sad(ness)' the definition 'to weep or make sad sounds' would be most relevant, but when generated in response to 'anger' the definition 'to call out/yell' was most appropriate (definitions taken from http://www.Thesaurus.com). Arguably participants may have had either meaning in mind when they generated the exemplar in response to the remaining emotion labels, which complicated the issue. We made the decision to merge 'cry' contingent on the first sadness-related definition, only, as the exemplar was most frequently given in response to the 'Sad' category. 'Cry' become the core action word, and 'weep' and 'sob' the subsidiary action words. As 'cry' was already the unmerged, top modal exemplar for 'sad(ness)', this merging decision did not change the modal response that was chosen for the 'sad' label in study 2. If we had alternatively (or additionally) chosen to merge according to the second definition, 'cry' could have been grouped with 'scream', 'shout' and 'shriek'. This was problematic as our criteria suggested that 'scream' and 'shriek' could be merged with 'yell', but 'yell' could not be merged with 'cry'. Therefore, the strategy adopted was both simpler, and more conservative.
} 
326 remaining responses). These exemplars are unlikely to represent words which commonly evoke

327 discrete affective knowledge; therefore, we decided to remove these responses from the sample

328 (see Buchanan et al., 2013). Following removal of idiosyncratic responses, there were 51

329 unique, modal action words; including 15 core action words, and 19 subsidiary action words.

330 Here 'modal' refers to an action word that was generated by two or more participants, but was

331 not synonymous with other responses and went unmerged. Therefore, they differ from 'core' and

332 'subsidiary' action words. This final selection represents $14 \%$ of the total number of unique

333 words originally generated.

334 The top three most frequently generated action words, per emotion label, are shown in Table 1.

335 Response frequencies are shown in parenthesis, in the second column. When these words

336 represent core exemplars, frequencies also include the number of participants who generated

337 subsidiary action words (corresponding subsidiary words are shown in the column three).

338 Frequencies above 25 are shown when a proportion of participants gave both the core and

339 subsidiary exemplars in response to the same emotion label. The full set of action words (core,

340 subsidiary and modal), are provided in Appendix A. In addition, all responses are provided in

341 the supplementary data file (acceptable and unacceptable idiosyncratic and modal responses).

[Insert Table 1 here]

346 Analysing by exemplar, $78.43 \%$ of all modal action words were generated in response to one

347 emotion label only, leaving $21.57 \%$ that were generated for multiple labels. This distinction was 
348 present even for the most frequently generated action words, displayed in Table 1 . When only

349 these exemplars were considered, $15.79 \%$ represented the most frequent responses for more than

350 one emotion label, and $68.75 \%$ were generated by at least two participants in response to one of

351 more other emotion labels. These findings support the work of Stevenson, Mikels and James,

352 (2007b). In their study, although $44.54 \%$ of ANEW words obtained ratings to suggest that they

353 were disproportionately associated with one (or two) discrete emotions, $22.70 \%$ of words were

354 associated with three or more emotion labels, representing an analogue to the 'overlapping'

355 exemplars in the present study.

356 Discussion

357 In the present study we introduced a constrained 'affective context' to identify action words that

358 were likely to evoke affective knowledge, proportionally relevant to different emotional states

359 (e.g. Stevenson, Mikels \& James, 2007a). The greater the number of participants that generated a

360 particular action word in response to an emotion label, the greater likelihood that that action

361 word would be situated in, and evoke affective knowledge relevant to that emotion. Both action

362 words and generation frequencies are available in Appendix A. We suggest possible uses for our

363 stimuli in the general discussion.

364 Importantly, findings suggest that participants generated a selection of action words that were

365 either strongly (or disproportionately) associated with a particular emotional state, or were

366 proportionally related to a number of different emotional states (overlapping exemplars). These

367 findings have important implications both for theories of affective, experiential grounding and

368 emotional attribution; the latter addressed in the general discussion. Some researchers suggest

369 that words are understood by evoking very specific representations of situations in which their

370 referents appear (e.g. Schwanenflugel \& Shoben, 1983; Pecher, Boot and van Dantzig, 2011). 
371 This parallels understanding of concrete concepts, which rely on reactivation in the same

372 sensorimotor areas initially recruited during interactions with the referent object (e.g. Barsalou,

373 1999). Finding that some action words were disproportionately associated with one emotion

374 label appear to provide support for this view. However, finding overlapping exemplars support

375 the notion that words are 'generally' evocative and have the potential to re-activate affective

376 knowledge relevant to a range of emotional states (e.g. Newcombe et al., 2012). In the present

377 study 'Cry' may be a particularly good example of a word that is 'generally' evocative. This

378 exemplar and its synonyms (e.g. sob and weep) were frequently given as exemplars in response

379 to the 'sad', 'anger' and 'fear' labels, and also by a smaller number of participants in response to

380 the 'happy' and 'surprise' labels. In study 2 we use a rating task to assess the robustness of the

381 most frequent action word-to-label associations, generated during study 1.

\section{Study 2- Validating associations between action words and emotion labels}

384 In study 2 we assess (a) the typicality of self-generated action words, and (b) the stability of

385 action word-to-label associations. We adopt a rating task, similar to Stevenson, Mikels and

386 James, (2007a), in which participants rate the relationship between the six most frequently

387 generated action words, and each discrete, emotion label. Emotion labels and action words are

388 presented within a sentence e.g. "if you see someone 'recoil' how likely are you to think that

389 they are feeling the following emotion?... 'disgust' ". Primarily, we would expect ratings to

390 indicate a comparatively stronger association between action words and the emotion labels to

391 which they were (most frequently) generated. This would confirm that the word is understood

392 due to its (dis)proportional activation of affective knowledge relevant to that emotional state. 
393 This validation attempt was particularly important for assessing whether the top exemplars 'cry'

394 and 'smile' were as strongly linked to the respective emotional states of 'sad(ness)' and

395 'happ(iness)' as generation frequencies suggested. This was a concern as both action word/label

396 pairs had been included as examples in the task instructions for study 1, so frequent endorsement

397 may not reflect spontaneous generation. This may also explain why the word 'cry' was given so

398 frequently, across the different 'affective contexts'. In addition, although participants were

399 discouraged from using the internet to generate their responses during study 1 , we were unable

400 to definitively rule out the possibility that they had done so. Use of external sources may have

401 inflated frequencies, artificially creating modal exemplars. Although this seems unlikely, as

402 participants generated a larger number of idiosyncratic than modal exemplars, it is important to

403 address this possible methodological issue.

404 Two further manipulations were applied to the rating task to test the robustness of action word-

405 to-label associations. First, we varied rating direction (i.e. whether participants made an action

406 word-to-emotion category, or emotion category-to-action word association). The following is an

407 example of an action word-to-category rating: "if you see someone 'cry', how likely are you to

408 think that they feel 'sad'?". Researchers commonly evaluate semantic relationships by

409 measuring both the 'forward' and 'backward' associations between category labels and

410 exemplars, and quantify the strength of the association using conditional probabilities (e.g.

411 Nelson, McEvoy \& Schreiber, 2004). Here conditional probabilities measure whether action

412 words evoke knowledge relevant to a particular emotional state as strongly as that emotional

413 state (label) evokes knowledge of the action word's referent.

414 Second, we asked participants to rate action word/category pairings from both a first person

415 perspective (e.g., "If you are 'crying', how likely is it that you are feeling 'sad'?") and a third 
416 person perspective. (e.g., “'if someone is 'crying', how likely are they to be feeling 'sad'?'). This

417 was an exploratory manipulation, which had the potential to inform us about the way in which

418 affective knowledge is used for emotional attribution. On the one hand, higher ratings between

419 action words and emotion labels might be expected when a first-person perspective is applied.

420 Given that affective knowledge is predominantly grounded in an individual's situated experience

421 (e.g. Vigliocco et al., 2009), words may preferentially evoke feelings that are self-relevant.

422 Conversely, participants may view a simpler correspondence between behaviours and emotions

423 for other people, than for themselves. Self-relevant affective knowledge may be richer and more

424 variable, complicating behaviour-to-state mappings when participants use first-person

425 instructions (e.g. 'people tend to act this way when they are feeling a certain emotion, but when I

426 was feeling happy I didn't act that way'). This account would predict stronger action word/label

427 ratings when participants adopt a third-person perspective.

428 Method

429 Ethics

430 This research is subject to ethical guidelines set out by the British Psychological Society (1993)

431 and was approved by the School of Psychology's ethics committee, at the University of Leeds

432 (reference number: 13-0032, date of approval: 24/02/2013). As before, informed consent items

433 were embedded in an online survey and participants agreed to take part by ticking a series of 434 boxes.

435 Design

436 A 2 (instruction perspective: first or third person, between) $\times 2$ (rating direction: category to

437 action word or action word to category, between) $\times 2$ (typicality: typical or atypical label/action 
438 word pairing, within) mixed factorial design was employed. The instruction perspective factor

439 manipulated whether participants received first-person perspective instructions ("if you are

440 feeling 'sad', how likely are you to act in the following way?" e.g. 'cry') or third person

441 perspective instructions ("if someone is feeling 'sad', how likely are they to act in the following

442 way?" e.g. 'cry'). The rating direction factor manipulated whether participants rated associations

443 in an action word-to-category direction ("if you are 'crying', how likely are you to be feeling the

444 following emotion?" e.g. 'sad') or a category-to-action word direction (“if you are feeling 'sad',

445 how likely are you to act in the following way" e.g. 'cry'). Participants each made 36 ratings,

446 based on all combinations of six discrete emotion labels and the action words most frequently

447 endorsed in response to each of these labels, during study 1. Feature generation data determined

448 whether emotion label / action word pairings were typical (e.g. six pairs, 'happy' and 'smile'), or

449 atypical (30 pairs, e.g. 'sad' and 'smile').

450 Participants were presented with an open-ended sentence for each rating, which included either

451 an emotion label or action-word e.g. "if you are feeling 'sad', how likely are you to act in the

452 following way?". Participants were invited to substitute each of the six action words (or labels)

453 into the end of this sentence (e.g. 'cry'), and to provide a likelihood rating for each label/action

454 word pairing. After all six ratings were submitted, participants were presented with the next

455 open-ended sentence, which included a new label (or action word). Overall, participants made

456 ratings in six, separate blocks, which presented a different label (or action word) to be rated

457 against each action word (or label), respectively. Block order was counterbalanced across

458 participants. Within a particular block, participants encountered each of the six ratings in a fixed

459 order. Although fixed per participant, this order was randomised per block, to ensure that the

460 typical pairing was not always presented in the same rating position (e.g. in the 'sad' block 
461 participants rated associations with action words in the following order: 'smile', 'cry' 'jump'...,

462 but in the 'happy' block they rated action words in a different order: 'hide', 'scream', 'smile'..)..

463 Therefore, while block order differed, rating order within blocks was the same for all

464 participants within a particular condition.

465 Participants

466 Forty participants each completed the task using first-person perspective instructions ( 25 female,

467 Mean age $=26.48, \mathrm{SD}=8.97)$ and third-person perspective instructions (29 female, Mean age $=$

$46827.53, \mathrm{SD}=9.47)$. Forty participants completed tasks that required category-to-action word

469 ratings $(31$ female, Mean age $=25.65, \mathrm{SD}=9.56)$ and forty completed tasks that required action

470 word-to-category ratings ( 29 female, Mean age $=28.35, \mathrm{SD}=8.70$ ).

471 Participants indicated whether they spoke any languages in addition to English and estimated

472 how many years they had been able to do so. Those judged to be fluent bilinguals or multi-

473 linguals were omitted from the sample. An opportunity recruitment method was used;

474 participants responded online, to links posted on social media sites (see Study 1). The study was

475 presented using the Survey Monkey platform (http://www.surveymonkey.com, Survey Monkey

476 Inc. Palo Alto, California, USA). There was no time limit imposed.

477 Materials

478 We re-used the six basic emotion labels from study 1 ('fear', 'happy', 'sad', 'disgust', 'anger'

479 and 'surprise', e.g. Ekman, 1992). The most frequently generated action words for each emotion

480 label were selected from the merged, feature generation data. They were as follows: 'scream'

481 (matched with 'anger'); 'smile' ('happy'), 'cry' ('sad'), 'recoil' ('disgust'), 'hide' ('fear') and

482 'jump' ('surprise'). 
483

484 485

486

487 488 and made 36 ratings in total.

\section{Procedure}

Each participant was randomly assigned to one of the four between-participants conditions of the 2 (instruction perspective) $\times 2$ (rating direction) design. Ratings were made on a five-point Likert-style scale for each question, anchored 'Very Unlikely' (1) to 'Very Likely' (5). All participants were presented with the same combination of emotion label/action word pairings

\section{Results}

\section{Data preparation}

491 For each emotion label, two mean ratings were calculated per participant. The 'typical' mean 492 was the rating given to the most typical label and emotion word pairing, according to the feature 493 generation data (e.g. 'cry' and 'sad'). The five remaining ratings given by the participant were 494 summed and then averaged to produce a grouped 'atypical' score (mean scores for the full set of 49536 label/action word ratings are shown in Appendix B).

496 Analysis

497 A 2 (instruction perspective: first or third) $\times 2$ (rating direction: category-to-action word or action word-to-category) $\times 6$ (category: sad, anger, happy, disgust, surprise, fear) $\times 2$ (typicality: typical or atypical) mixed factorial ANOVA was performed. Instruction perspective and rating

500 direction were between-subjects factors. Main effects and interactions are displayed in Table 2.

501 Hereafter we focus on interactions with the typicality factor. 'Typicality' reflects the strength of

502 association between action words and emotion labels (operationalised here as high or low), thus

503 indicating the likelihood that action words disproportionately evoke affective knowledge 504 relevant to emotional states. 
Validation of self-generation data:

510 Participants gave significantly higher mean likelihood ratings to typical pairings ( $\mathrm{M}=4.31, \mathrm{SD}$

$511=0.56)$, than grouped atypical pairings, $(\mathrm{M}=2.56, \mathrm{SD}=0.49)$, on a scale from $1-5$. This finding

512 provides support for the label-action word associations derived from the feature generation data

513 (study 1). The typicality effect was qualified by a significant interaction with emotion category,

514 prompting investigation of the effect for each discrete, emotion category (see Table 3 and Figure $5151)$.

517 All six paired samples t-tests were significant and in the anticipated direction (typical

518 category/action word pairings received higher association ratings than the grouped atypical

519 pairings). Therefore, the interaction likely reflects general differences in the strength with which

520 typical action words evoke affective knowledge disproportionally related to corresponding

521 emotional states, all effects being conventionally large $(d>0.8)$. In support, Figure 1 shows that

522 the $95 \%$ confidence intervals for mean typical ratings and the summed average of atypical

523 ratings did not overlap for any emotion category. That typicality predictions were supported

524 weakens the suggestion that participants used the internet to generate their responses during

525 study 1. In addition, typicality effects were present for the specific pairings of 'happy' / 'smile' 
526 and 'sad' / 'cry' pairings, reducing the likelihood that participants generated these associations

527 simply as a result of their inclusion in previous task instructions.

528

529

[Insert Figure 1 here]

530

531 Further manipulations and typicality ratings.

532 Rating Direction

533 The typicality $\times$ rating direction $\times$ emotion category interaction was significant. Separate

534 typicality $\times$ rating direction analyses were conducted for each emotion category (see Table 4 ).

[Insert Table 4 here]

537

538 There were significant typicality $\times$ direction rating interactions for the 'sad' and 'fear'

539 categories.

540 Interactions followed a similar pattern for both emotion categories. As predicted, paired samples

541 t-tests showed that participants gave significantly higher likelihood ratings to typical vs. atypical

542 pairs, for both action word-to-category pairings $\left[t_{\text {sad }}(39)=24.12, p<0.001, d=5.33\right.$; $t_{\text {fear }}(39)=$

$54312.74, p<0.001, d=2.30]$, and category-to-action word pairings $\left[t_{\text {sad }}(38)=13.34, p<0.001, d\right.$

$\left.544=2.51 ; t_{\text {fear }}(38)=6.98, p<0.001, d=1.10\right]$. Independent samples t-tests showed that

545 participants rated atypical pairs similarly, independent of rating direction, $\left[t_{\text {sad }}(74)=-0.079, p=\right.$

$\left.5460.94 ; t_{\text {fear }}(84)=-0.16, p=0.88\right]$, but gave significantly higher ratings to typical pairings 
547 presented in an action word-to-category format than a category-to-action word format, $\left[t_{\text {sad }}(84)=\right.$

$\left.548-2.06, p=0.043, d=0.68 ; t_{\text {fear }}(84)=-2.004, p=0.048, d=0.59\right]$. In sum, for 'Fear' and 'Sad'

549 categories, typical pairings were given comparatively higher likelihood ratings when rated in an

550 action word-to-category vs. category-to-action word direction.

551

552 Instruction perspective

553 Critically, there were no significant instruction perspective $\times$ typicality, or instruction

554 perspective $\times$ typicality $\times$ category interactions $(\mathrm{p}>0.10)$.

555 However, the instruction perspective $\times$ rating direction $\times$ typicality $\times$ category interaction was

556 significant (see descriptive statistics in Table 5).

560 To explore this interaction, separate instruction perspective $\times$ typicality $\times$ direction rating mixed

561 factorial ANOVAs were conducted for each emotion category. There was a significant

562 interaction for one category only: 'disgust'; $F(1,82)=8.71, \mathrm{MSe}=0.79, p=0.004, \eta_{p}{ }^{2}=0.097^{5}$.

5 To explore this interaction, separate direction rating $\times$ typicality mixed ANOVAs were conducted for disgust ratings, for participants who received first and third person instructions, respectively. This two-way interaction was significant for participants who received first-person instructions, $F(1,37)=13.06$, MSE $=0.65, p=0.001, \eta_{p}{ }^{2}=0.26$, but not for those who received third person instructions, $F(1,37)=0.45$, MSE $=0.93, p=0.51, \eta_{p}{ }^{2}=0.012$.

Paired samples t-tests revealed that, independent of direction rating, participants who had received first person instructions always gave higher ratings to the typical pairing, than grouped atypical pairings, $\left[t_{\text {category-to-action }} \operatorname{word}(18)=3.90, p=0.001, d=1.20 ; t_{\text {action word-to-category }}(19)=12.13, \mathrm{p}<\right.$ $0.001, \mathrm{~d}=3.37$. $]$ While independent $\mathrm{t}$-tests showed that these participants rated atypical pairings similarly in both rating directions $[t(37)=1.84$, $p=0.074$, they gave significantly higher ratings to the typical pairing when embedded in action word-to-category versus category-to-action word sentences, $t(37)=2.70, p=0.010, d=0.89$. 
Discussion

565 Ratings confirm that participants were more likely to associate action words with the emotional

566 state to which they had been typically generated in study 1 . This lessens the likelihood that

567 endorsement was inflated by the examples included in task instructions, or use of the internet. In

568 addition, direction and person perspective manipulations had little impact on ratings, indicating

569 that typical pairings contained action words and emotional states that were robustly associated.

570 Overall, these findings validate the associations derived during study 1 and support the notion

571 that action word meaning is proportionally grounded in, and evokes affective knowledge

572 relevant for different emotional states.

573 However, it is important to acknowledge the following issue: task design meant that participants

574 rated one label (or action word) in association with all six action words (or labels) before they

575 were presented with the next label (or action word). This may have encouraged participants to

576 adopt a 'relative' rating strategy, in which they simultaneously compared the likely association

577 between all six items and the dominant label, or action word. Typical pairings may then receive

578 the highest likelihood ratings because they represent the 'best option', rather than giving a true

579 indication of the way in which action words proportionally activate affective knowledge relevant

580 to the presented label. This limitation is compounded as, per block, participants responded to the

581 six pairings in the same order. Any biases that this presentation strategy encouraged would

582 therefore be applicable to all participants, despite care to vary presentation of the typical pair, per

583 block.

584 However, our data suggest it is unlikely that participants automatically employed a comparative

585 rating strategy. If they had we would expect all atypical pairings to receive very low ratings on 
586 the scale. Although some of the averaged, atypical ratings were below the scale midpoint (2.5;

587 'happy', 'anger', 'sad' and 'disgust'), others were higher ('fear' and 'surprise'). These findings

588 are expected given that there were overlaps in the some of the typical action words included in

589 the task and the top, three modal action words generated for other emotion labels, during study 1.

590 This was the case for the three labels that attracted the highest average atypical ratings ('fear',

591 'surprise' and 'anger'). For example, although the action word 'cry' represented the typical

592 exemplar for the label 'sad', it was also frequently generated in response to the emotion labels

593 'fear' and 'anger' (see table 1). Similarly, the typical action word for the label 'anger' ('scream')

594 had been frequently endorsed in response to the label 'surprise'. The inclusion of these

595 overlapping exemplars meant that, for some emotion labels, not all 'atypical' exemplars were

596 equally 'atypical', inflating the averaged atypical ratings. Importantly, these findings indicate

597 that participants judged each action word/label pair based on the 'absolute' association between

598 the two words, rather than making a comparative judgment that was biased by the presence of an

599 obviously 'typical' pairing. They also support the idea that 'typicality' is expressed as a matter

600 of degree, as action words may simultaneously evoke affective knowledge relevant to several

601 emotional states (e.g. Newcombe et al., 2012).

602 One further finding should be highlighted. When direction was manipulated, ratings revealed

603 different forward and backward connection strengths between the emotion labels 'fear' and 'sad'

604 and their paired, typical action words. In both cases participants gave higher ratings when

605 presented with the pair in action word-to-category order, than in category-to-action word order

606 (e.g. $\mathrm{P}($ Hide $\mid$ Fear $)<\mathrm{P}($ Fear $\mid$ Hide $)$. This trend was also present for the label 'disgust' and typical

607 action word of 'recoil', but only when the pairing was considered from a first-person perspective 608 (see footnote 4). 
609 To aid interpretation we explicitly consider the behaviours to which action words refer, and how

610 they may inform emotional attribution. The present data suggest that the propensity to 'hide'

611 ('cry') when expressing 'fear' ('sadness') may vary depending on the type of stimulus causing

612 'fear' ('sadness'), but that given the behaviour of hiding (crying), the likelihood that a person is

613 experiencing fear (sadness) is much greater. Arguably the latter attributional pattern may be

614 more prevalent in Western societies. Here people are often encouraged to mask or regulate

615 behavioural signs of emotional states that cause them to be perceived as weak in public, like

616 'sadness' and 'fear' (e.g. Wierzbicka, 1994; Barrett, Mesquita \& Gendron, 2011). If related

617 behaviours are observed then the attribution process may be more automatic. A justification may

618 follow: 'I/they must be feeling very 'sad' if I/they feel the need to 'cry' in public.' In sum, while

619 the present data confirm that there is stability in the way some action words disproportionately

620 evoke affective knowledge, there is some evidence that cultural background may influence the

621 way affective knowledge is constructed and used for attribution (e.g. Barrett, Mesquita \&

622 Gendron, 2011).

\section{General Discussion}

624 We provide a set of emotion-related action words, accompanied by data to show how strongly

625 each word evokes emotional knowledge relevant to several, discrete emotional states. This work

626 is consistent with the proposal that emotion words are grounded in affective knowledge (e.g.

627 Vigliocco et al., 2009) and complements previous research, by exploring whether word-to-

628 knowledge links are constructed, at least partially, in a categorical fashion (e.g. Stevenson,

629 Mikels \& James, 2007a; Briesemeister, Kuchinke \& Jacobs, 2011a; 2011b, 2014; Westbury et

630 al., 2014). 
631 Action words were elicited from participants using a constrained feature-generation task (e.g.

632 McRae et al., 2005; Vinson \& Vigliocco, 2008). Emotion labels were used to create (and

633 constrain) six, different 'affective contexts' (e.g. Stevenson, Mikels \& James, 2007a;

634 Briesemeister, Kuchinke \& Jacobs, 2011a). This method allowed assessment of the strength with

635 which each action word elicited specific affective knowledge; the larger the number of

636 participants who endorsed the pair the greater the likelihood that the word (dis)proportionally

637 evoked knowledge relevant to that emotional state. Using a rating task (study 2) we confirmed

638 that the action words most frequently elicited in study 1 were more likely to be associated with

639 the emotion label to which they had been generated (typical pairs), than to other emotion labels

640 (atypical pairs). Typical pairs also retained rating dominance when two further sentence-based

641 manipulations were applied (rating direction and person perspective), suggesting a degree of

642 robustness in the way typical words evoke affective knowledge.

643 To facilitate use of the current stimuli, all acceptable action words, generated by two or more

644 participants in study 1, are included in Appendix A (a fuller list, including idiosyncratic

645 responses, is provided in the supplementary materials). Words are presented alongside raw,

646 unmerged frequencies to indicate the number of participants who generated the action word in

647 response to each emotion label. This will allow researchers to select stimuli, based on unmerged

648 frequencies, or apply their own merging criteria. However, for completeness, we also indicate

649 whether the action word was classed as a 'core', 'subsidiary' (i.e. a synonym for the selected

650 'core' exemplar) or modal exemplar (a unique, non-synonymous response), based on our

651 merging criteria. Further, we provide ratings for each of the 36 action word/label pairs, included

652 in study 2 (Appendix B). 
653 On the one hand the current approach, and data produced, may provide an alternative way to

654 select emotional stimuli, based on the extent to which each word is likely to evoke specific

655 affective knowledge (e.g. Stevenson, Mikels \& James, 2007a; Briesemeister, Kuchinke \&

656 Jacobs, 2014). The current set of action words may be highly compatible for particular types of

657 task. Previous research shows that participants mimic congruent facial expressions when they

658 encounter emotion words (e.g. Foroni \& Semin, 2009), and that mimicry leads to enhanced

659 processing of subsequently presented emotional stimuli e.g. valence-congruent sentences (e.g.

660 Havas, Glenberg \& Rinck, 2006) and facial expressions (e.g. Halberstadt et al., 2009). Based on

661 Vigliocco et al's., (2009) framework, we might expect emotion-related action words to more

662 strongly elicit congruent facial mimicry, given their dual grounding in affective (Vinson, Ponari

663 \& Vigliocco, 2014) and sensorimotor knowledge (e.g. Hauk, Johnsrude \& Pulvermüller, 2004;

664 Niedenthal et al., 2009; Moseley et al., 2012). However, few studies incorporate action words

665 and those that do find inconsistent evidence for a verb (vs. adjective) advantage (Foroni \&

666 Semin, 2009; Halberstadt et al., 2009). If these findings reflect inconsistent use of linguistic

667 stimuli then our data may help by providing a larger set to select from. Further, by choosing

668 words that are both disproportionally related to a particular emotional state and related to facial

669 actions, researchers may extend investigations into whether language-mediated facial mimicry is

670 'category' or 'valence' driven. Specifically, whether reading an action word strongly associated

671 with 'fear' specifically induces mimicry in category-diagnostic features of a fearful face, (Ponari

672 et al., 2012) or whether reading any negatively valenced word induces a similar pattern of

673 negative mimicry.

674 On the other hand, the present data may encourage two types of 'additive' approach, important

675 for assessing the relative validity of current attempts to measure affective grounding (e.g. 
676 Newcombe et al., 2012). First, as we provide new categorical data for words which already have

677 dimensional rating norms (e.g. Warriner, Kuperman \& Brysbaert, 2013), we facilitate attempts to

678 assess whether categorical and dimensional ratings are mutually predictive of one another, or

679 quantify emotional information in the same way. Stevenson, Mikels and James (2007a) and

680 Westbury et al., (2014) have conducted similar work, both showing a degree of heterogeneity in

681 the ability of categorical ratings to predict dimensional ratings. In particular, Westbury et al.,

682 (2014) showed that co-occurrence distances between emotion labels and words were more

683 strongly predictive of valence, than arousal ratings, and that both types of dimensional rating

684 were predicted by co-occurrence distances from distinct sets of emotion labels (e.g. those

685 naming 'automatic' emotions, like 'panic', for arousal, and those associated with approachability

686 and potency, for valence).

687 This approach could also be used to assess the relationships between the current categorical data

688 and semantic richness norms (e.g. emotional experience ratings), which assess the magnitude to

689 which words evoke undifferentiated, affective knowledge (e.g. Newcombe et al., 2012). This is

690 not yet possible, as Newcombe et al., (2012) only provide normative data for nouns. It would be

691 particularly interesting to provide a comparison for overlapping exemplars, such as 'cry', which

692 our participants modally endorsed as evoking affective knowledge relevant to five of the six

693 discrete emotional states. We might expect emotional experience ratings to fluctuate dependent

694 on both the number of emotional states that the word can be associated with, and the frequency

695 of endorsement, across emotions.

696 A second, related investigation, would involve entering different types of rating as separate

697 predictors, to assess whether they account for unique variance in emotional word processing

698 outcomes. Previous work focuses on prediction of lexical decision latencies, presumably because 
699 large datasets of reaction times already exist (e.g. Balota et al., 2007; Keuleers et al., 2012).

700 However, it may be equally possible to apply ratings as predictors to other types of task that

701 examine emotional word processing (Briesemeister, Kuchinke \& Jacobs, 2011b). For example,

702 the emotional Stroop task (Mackay et al., 2004) and De Houwer's (2003) affective Simon task

703 (Altarriba \& Basnight-Brown, 2010). So far, lexical decision data confirm that categorical and

704 dimensional ratings account for unique variance and that, when combined, ratings account for a

705 slightly larger proportion of overall variance in latencies than they do independently (e.g.

706 Briesemeister, Kuchinke \& Jacobs, 2011a; 2011b; 2014; see also Newcombe et al., 2012 and

707 Moffat et al., 2015 for comparisons of semantic richness and dimensional ratings). In support,

708 physiological evidence shows that both types of information are important for emotion word

709 processing; when words are disproportionately associated with particular emotional states then

710 categorical information is processed first, followed by dimensional or valence-based properties

711 of the word (e.g. Briesemeister, Kuchinke \& Jacobs, 2014; Briesemeister et al., 2014). Linear

712 processing stages are consistent with Panksepp's (1998; 2012) hierarchical model, which

713 includes a secondary, automatic stage for categorical processing of emotional stimuli (relation to

714 the proposed play, seeking, rage, lust, fear, panic and care subsystems), and a subsequent,

715 tertiary stage, in which dimensional properties of the stimuli are considered.

716 However, one caveat is important when considering the compatibility of our stimuli for lexical

717 decision, or other tasks that require single-word processing. As previously argued, participants

718 tend to be poor or inconsistent in their ability to extract affective meaning from verbs (e.g.

719 Schacht \& Sommer, 2009; Palazova et al., 2011). Meaning activation will depend on the task in

720 which the verb is presented, and its associated goals. For example, when action words are presented in

721 isolation and participants make a non-affective judgment, as they do in lexical decision tasks, action 
722

723

724

725

726

727

728

729

730

731

732

733

734

735

736

737

738

739

740

741

742

743

744

words are unlikely to spontaneously evoke the same constrained, affective knowledge that they do in the

present work. Therefore, in order to assess whether categorical ratings predict action word

processing, the same 'affective context' might need to be applied to the new task. Following

Schacht and Sommer's (2009) approach, researchers might present the word pair 'sad' and 'cry', asking participants to respond to the action word in the pair, only.

Situated approaches emphasise that words are referents for experiential components; in this case, behaviours. As such, some of our findings have implications for how overt cues influence emotional attribution and interpretation. Finding that participants sometimes associated the same behaviours with several emotional states in study 1 , and showed fluctuations in their ratings of atypical behaviour/state pairings in study 2, both stand in contrast to basic emotion views (e.g. Ekman, 1992). These accounts suggest that behaviours show strong, discrete, relationships with basic emotional states and are important diagnostic cues for interpretation. In contrast, 'proportional' associations are favoured both by construction and componential models (e.g. Scherer, 1984; Smith \& Ellsworth, 1985; Barrett, Lindquist \& Gendron, 2007; Lindquist, 2009). According to these accounts, behavioural cues need not be diagnostic as emotional interpretation is driven by the summation of multiple pieces of evidence, only some of which will be present at the time of perception (e.g. Smith \& Ellsworth, 1985; Lindquist \& Gendron, 2013). People flexibly recruit other 'evidence' from a highly intra-individual repository of affective knowledge, built through relevant past and present experiences (e.g. what precipitated the current emotional state, how the actor has behaved in the past, how the observer themselves felt under similar circumstances). Some of this knowledge will be shaped by the societal or cultural norms applicable to the individual (see study 2, e.g. Barrett, Mesquita \& Gendron, 2011).

Flexible knowledge recruitment explains why the same behaviour may be interpreted to 
745 represent different emotional states by different observers, or by the same observer, across

746 different time-points (e.g. Lindquist \& Gendron, 2013).

747 In conclusion, we provide a set of English action words, characterised by their proportional

748 likelihood to evoke affective knowledge relevant to different emotional states. We used basic

749 emotion labels to create a set of constrained 'affective contexts', both for initial generation of

750 action words (study 1) and validation of the most typical exemplars (study 2). Our stimuli both

751 complement and extend existing linguistic databases that contain categorical norms (e.g.

752 Stevenson, Mikels \& James, 2007a; Briesemeister, Kuchinke \& Jacobs, 2011a). Our data may

753 similarly be used to explore whether emotional word processing is predicted by categorical

754 norms alone, or in conjunction with other types of rating (e.g. dimensional or semantic richness

755 ratings, Lang, 1980; Newcombe et al., 2012).

756

757

758

759

760

761

762

763

764

765 
References

768

769

770

771

772

773

774

775

776

777

778

779

780

781

782

783

784

785

Altarriba, J., \& Basnight-Brown, D. M. (2010). The representation of emotion vs. emotion-laden words in English and Spanish in the Affective Simon Task. International Journal of Bilingualism, 15:310-328.

Balota, D. A., Yap, M. J., Cortese, M. J., Hutchison, K. A., Kessler, B., Loftis, B., Neely, J. H., Nelson, D. H., Simpson, G. B., \& Treiman, R. (2007). The English Lexicon Project. Behavior Research Methods, 39(3): 445-459.

Barrett, L. F., Lindquist, K. A., \& Gendron, M. (2007). Language as context for the perception of emotion. Trends in Cognitive Sciences, 11(8):327-332.

Barrett, L. F., Mesquita, B., \& Gendron, M. (2011). Context in emotion perception. Current Directions in Psychological Science, 20(5):286-290.

Barsalou, L. W. (1999). Perceptual symbol systems. Behavioral and Brain Sciences, 22: $577-$ 660.

Barsalou, L. W., A. Santos, W. K. Simmons \& C. D. Wilson. (2008). Language and simulation in conceptual processing. In M. De Vega, A. M. Glenberg \& A. C. Graesser (eds.), Symbols, embodiment, and meaning. Oxford: Oxford University Press.

Bradley, M. M., \& Lang, P. J. (1999). Affective Norms for English Words (ANEW): Instruction manual and affective ratings (Technical Report C-1) The Center for Research in Psychophysiology. Gainesville: University of Florida. 
786 787

788 789

790

791

792

793

794

795

796

797

798

799

800

801

802

803

804

805

Briesemeister, B. B., Kuchinke, L., \& Jacobs, A. M. (2011a). Discrete emotion norms for nouns: Berlin affective word list (DENN-BAWL). Behavior Research Methods, 43(2):441-448.

Briesemeister, B. B., Kuchinke, L., \& Jacobs, A. M. (2011b). Discrete emotion effects on lexical decision response times. PloS one, 6(8):e23743.

Briesemeister, B. B., Kuchinke, L., \& Jacobs, A. M. (2014). Emotion word recognition: Discrete information effects first, continuous later? Brain research, 1564:62-71.

Briesemeister, B. B., Kuchinke, L., Jacobs, A. M., \& Braun, M. (2014). Emotions in reading: Dissociation of happiness and positivity. Cognitive, Affective, \& Behavioral Neuroscience, 15, 287-298.

Buchanan, E. M., Holmes, J. L., Teasley, M. L., \& Hutchison, K. A. (2013). English semantic word-pair norms and a searchable Web portal for experimental stimulus creation. Behavior Research Methods, 45(3): 746-757.

De Houwer, J. (2003). The extrinsic affective Simon task. Experimental Psychology, 50(2):7785.

Doost, H. T. N., Moradi, A. R., Taghavi, M. R., Yule, W., \& Dalgleish, T. (1999). The development of a corpus of emotional words produced by children and adolescents. Personality and Individual Differences, 27(3):433-451.

Ekman, P. (1992). An argument for basic emotions. Cognition \& Emotion, 6(3-4):169-200.

Estes, Z., \& Adelman, J. S. (2008). Automatic vigilance for negative words is categorical and general. Emotion, 8:453-457 
806 Foroni, F., \& Semin, G. R. (2009). Language that puts you in touch with your bodily feelings:

807 The multimodal responsiveness of affective expressions. Psychological

$808 \quad$ Science, 20(8):74-980.

809 Frijda, N. H. (1986). The Emotions. Cambridge, UK: Cambridge University Press.

810 Frijda, N. H., Kuipers, P., \& Ter Schure, E. (1989). Relations among emotion, appraisal, and 811 emotional action readiness. Journal of Personality and Social Psychology, 57(2):212-

812228.

813 Halberstadt, J., Winkielman, P., Niedenthal, P. M., \& Dalle, N. (2009). Emotional conception

814 how embodied emotion concepts guide perception and facial action. Psychological

$815 \quad$ Science, 20(10):1254-1261.

816 Hauk, O., I. Johnsrude \& F. Pulvermüller. (2004). Somatotopic representation of actions words

817 in human motor and premotor cortex. Neuron, 41:301-307

818 Havas, D. A., Glenberg, A. M., \& Rinck, M. (2007). Emotion simulation during language

819 comprehension. Psychonomic Bulletin \& Review, 14(3):436-441.

820 Herbert, C., Junghöfer, M., \& Kissler, J. (2008). Event related potentials to emotional adjectives 821 during reading. Psychophysiology, 45(3):487-498.

822 Herbert, C., Kissler, J., Junghöfer, M., Peyk, P., \& Rockstroh, B. (2006). Processing of 823 emotional adjectives: Evidence from startle EMG and ERPs. Psychophysiology, 43:197824206.

825 Hutchison, K. A., Balota, D. A., Cortese, M. J., Neely, J. H., Niemeyer, D., Bengson, J. J., \&

826 Cohen-Shikora, E. R., Tse, C-S., Yap, M. J., Bengson, J. J., Niemeyer, D. \& Buchanan, 

E. (2010). The Semantic Priming Project. Behavior Research Methods, 45(4): 10991114.

829 Kanske, P., \& Kotz, S. A. (2007). Concreteness in emotional words: ERP evidence from a $830 \quad$ hemifield study. Brain research, 1148: 138-148.

831 Keuleers, E., Lacey, P., Rastle, K., \& Brysbaert, M. (2012). The British Lexicon Project: Lexical 832 decision data for 28,730 monosyllabic and disyllabic English words. Behavior Research 833 Methods, 44(1): 287-304.

834 Kissler, J., Herbert, C., Peyk, P., \& Junghöfer, M. (2007). Buzzwords-Early cortical responses 835 to emotional words during reading. Psychological Science, 18(6):475-480.

836 Kousta, S. T., Vigliocco, G., Vinson, D. P., Andrews, M., \& Del Campo, E. (2011). The 837 representation of abstract words: why emotion matters. Journal of Experimental 838 Psychology: General, 140(1):14-34

839 Kousta, S. T., Vinson, D. P., \& Vigliocco, G. (2009). Emotion words, regardless of polarity, 840 have a processing advantage over neutral words. Cognition, 112(3): 473-481.

841 Kuperman, V. (2014). Virtual experiments in megastudies: A case study of language and 842 emotion. The Quarterly Journal of Experimental Psychology, DOI:

843 10.1080/17470218.2014.989865.

844 Kuperman, V., Estes, Z., Brysbaert, M., \& Warriner, A. B. (2014). Emotion and language: 845 Valence and arousal affect word recognition. Journal of Experimental Psychology:

846 General, 143(3): 1065-1081. 
847 Lang, P. J. (1980). Behavioral treatment and bio-behavioral assessment: computer applications.

848 In J. B. Sidowski, J. H. Johnson, \& T. A. Williams (Eds.), Technology in mental health 849 care delivery systems (pp. 119-137). Norwood, NJ: Ablex.

850 Larsen, R. J., Mercer, K. A., Balota, D. A., \& Strube, M. J. (2008). Not all negative words slow 851 down lexical decision and naming speed: Importance of word arousal. Emotion, 8: 445$852 \quad 452$

853 Lindquist, K. A. (2009). Language is powerful. Emotion Review, 1(1):16-18.

854 Lindquist, K. A., \& Gendron, M. (2013). What's in a word? Language constructs emotion 855 perception. Emotion Review, 5(1):66-71.

856 MacKay, D. G., Shafto, M., Taylor, J. K., Marian, D. E., Abrams, L., \& Dyer, J. R. (2004).

857 Relations between emotion, memory, and attention: Evidence from taboo Stroop, lexical 858 decision, and immediate memory tasks. Memory and Cognition, 32: 474-488

859 McEvoy, C. L., \& Nelson, D. L. (1982). Category name and instance norms for 106 categories 860 of various sizes. The American Journal of Psychology, 95(4): 581-634.

861 McRae, K., Cree, G. S., Seidenberg, M. S., \& McNorgan, C. (2005). Semantic feature 862 production norms for a large set of living and non-living things. Behavior research $863 \quad$ methods, 37(4): 547-559.

864 Moffat, M., Siakaluk, P. D., Sidhu, D. M., \& Pexman, P. M. (2014). Situated conceptualization 865 and semantic processing: effects of emotional experience and context availability in 866 semantic categorization and naming tasks. Psychonomic Bulletin \& Review, 22: 408-419.

867 Moseley, R., Carota, F., Hauk, O., Mohr, B., \& Pulvermüller, F. (2012). A role for the motor 868 system in binding abstract emotional meaning. Cerebral Cortex, 22: 1634-1647. 
869 Nelson, D. L., McEvoy, C. L., \& Schreiber, T. A. (2004). The University of South Florida free association, rhyme, and word fragment norms. Behavior Research Methods, Instruments, \& Computers, 36(3):402-407.

872 Newcombe, P. I., Campbell, C., Siakaluk, P. D., \& Pexman, P. M. (2012). Effects of emotional 873 and sensorimotor knowledge in semantic processing of concrete and abstract nouns. Frontiers in Human Neuroscience, 6: A275.

875 Niedenthal, P. M., Winkielman, P., Mondillon, L., \& Vermeulen, N. (2009). Embodiment of emotion concepts. Journal of Personality and Social Psychology, 96(6), 1120-1136.

Panksepp, J. (1998). Affective neuroscience: The foundations of human and animal emotions. New York, NY: Oxford University Press.

879 Panksepp, J. (2012). What is an emotional feeling? Lessons about affective origins from cross880 species neuroscience. Motivation and Emotion, 36: 4-15.

881

882

883

884

885

886

887

888

Palazova, M., Mantwill, K., Sommer, W., \& Schacht, A. (2011). Are effects of emotion in single words non-lexical? Evidence from event-related brain potentials. Neuropsychologia, 49(9): 2766-2775.

Palazova, M., Sommer, W., \& Schacht, A. (2013). Interplay of emotional valence and concreteness in word processing: An event-related potential study with verbs. Brain and Language, 125(3): 264-271.

Pavlenko, A. (2008). Emotion and emotion-laden words in the bilingual lexicon. Bilingualism: Language and Cognition, 11(2):147-164. 
889 Pecher, D., Boot, I., \& van Dantzig, S. (2011). Abstract concepts: Sensory-motor grounding, 890 metaphors, and beyond. In B. Ross (Ed.), The Psychology of Learning and Motivation

891 (Vol. 54) (pp. 217-248). Burlington: Academic Press.

892 Ponari, M., Conson, M., D'Amico, N. P., Grossi, D., \& Trojano, L. (2012). Mapping

893 correspondence between facial mimicry and emotion recognition in healthy

894 subjects. Emotion, 12(6):1398-1403

895

Pulvermüller, F. (1999). Words in the brain's language. Behavioral and Brain

896 Sciences, 22(2):253-279.

897

898

899

900

901

902

903

904

905

906

907

908

909

910

Schacht, A., \& Sommer, W. (2009). Time course and task dependence of emotion effects in word processing. Cognitive, Affective, \& Behavioral Neuroscience, 9(1):28-43.

Scherer, K. R. (1984) On the nature and function of emotion: a component process approach. In K. R. Scherer \& P. Ekman (Eds.). Approaches to Emotion (pp. 293-317). Hillsdale, NJ: Erlbaum.

Schock, J., Cortese, M. J., \& Khanna, M. M. (2012). Imageability estimates for 3,000 disyllabic words. Behavior Research Methods, 44(2): 374-379.

Schwanenflugel, P. J., \& Shoben, E. J. (1983). Differential context effects in the comprehension of abstract and concrete verbal materials. Journal of Experimental Psychology: Learning, Memory, and Cognition, 9(1): 82-102.

Shaoul, C., \& Westbury, C. (2010). Exploring lexical co-occurrence space using HiDEx. Behavior Research Methods, 42: 393-413.

Siakaluk, P. D., Knol, N., \& Pexman, P. M. (2014). Effects of emotional experience for abstract words in the Stroop task. Cognitive Science, 38(8): 1698-1717. 
911 Siakaluk, P. D., Pexman, P. M., Aguilera, L., Owen, W. J., and Sears, C. R. (2008). Evidence for 912 the activation of sensorimotor information during visual word recognition: the body913 object interaction effect. Cognition, 106: 433-443.

914 Smith, C. A., \& Ellsworth, P. C. (1985). Patterns of cognitive appraisal in emotion. Journal of 915 Personality and Social Psychology, 48(4):813-838.

916 Stevenson, R. A., Mikels, J. A., \& James, T. W. (2007a). Characterization of the affective norms 917 for English words by discrete emotional categories. Behavior Research 918 Methods, 39(4):1020-1024.

919 Stevenson R. A., Mikels J. A., James T. W. (2007b). Manual for the categorization of the 920 Affective Norms for English Words (ANEW) by discrete emotions.

921 Available:http://www.indiana.edu/_panlab/people/ras/Stevenson_ANEWManual_2007.

922 pdf. 12 May, 2015.

923 Vigliocco, G., Meteyard, L., Andrews, M., \& Kousta, S. (2009). Toward a theory of semantic 924 representation. Language and Cognition, 1(2):219-247.

925 Vinson, D., Ponari, M., \& Vigliocco, G. (2014). How does emotional content affect lexical 926 processing? Cognition \& Emotion, 28(4):737-746.

927 Vinson, D. P., \& Vigliocco, G. (2008). Semantic feature production norms for a large set of 928 objects and events. Behavior Research Methods, 40(1): 183-190.

929 Võ, M. L., Jacobs, A. M., \& Conrad, M. (2006). Cross-validating the Berlin affective word 930 list. Behavior Research Methods, 38(4):606-609. 
931 Võ, M. L., Conrad, M., Kuchinke, L., Urton, K., Hofmann, M. J., \& Jacobs, A. M. (2009). The 932 Berlin affective word list reloaded (BAWL-R). Behavior Research Methods, 41(2):534933538.

934 Warriner, A. B., Kuperman, V., \& Brysbaert, M. (2013). Norms of valence, arousal, and 935 dominance for 13,915 English lemmas. Behavior Research Methods, 45: 1191-1207.

936 Westbury, C., Keith, J., Briesemeister, B. B., Hofmann, M. J., \& Jacobs, A. M. (2014). Avoid 937 violence, rioting, and outrage; approach celebration, delight, and strength: Using large

940 Wiemer-Hastings, K., \& Xu, X. (2005). Content differences for abstract and concrete

941 concepts. Cognitive Science, 29(5):719-736.

942 Wierzbicka, A. (1994) Emotion, language and cultural scripts. In S. Kitayama \& H. R. Markus (Eds.). Emotion and Culture: Empirical studies of mutual influence (pp. 133-196). Washington, DC: American Psychological Association.

945 Wilson-Mendenhall, C. D., Barrett, L. F., Simmons, W. K., \& Barsalou, L. W. (2011). Grounding emotion in situated conceptualization. Neuropsychologia, 49(5): 1105-1127 


\section{Table $\mathbf{1}_{\text {(on next page) }}$}

Top three, most frequently generated action words for each emotion label

Top three, most frequently generated action words for each emotion label. Action words are presented alongside subsidiary responses (where appropriate). Response frequencies for each action word are presented within parenthesis in the second column. These frequencies represent merged totals when a corresponding subsidiary action word is shown in the third column. 
2 Table 1: Top three, most frequently generated action words for each emotion label, presented alongside subsidiary responses (where 3 appropriate). Response frequencies for each action word are presented within parenthesis in the second column. These frequencies represent 4 merged totals when a corresponding subsidiary action word in shown in the third column.

5

\begin{tabular}{lll}
\hline Emotion Label & $\begin{array}{l}\text { Most frequent action words (response } \\
\text { frequency) }\end{array}$ & Corresponding, subsidiary action words (core action word) \\
\hline Anger & Scream (34); Hit (13); Cry (7) & Shout/Yell/Shriek (scream); punch (hit); sob/weep (cry) \\
Happy & Smile (27); Laugh (20); Dance (10) & Grin (smile); Giggle (laugh); Skip (dance) \\
Sad & Cry (23); Frown (9), Withdraw (7) & Sob/Weep (cry); Grimace (Frown) \\
Disgust & Recoil (7); Frown (6); Gag/Vomit (5 each) & Cringe (Recoil); Grimace (Frown); Retch (Gag) \\
Fear & Hide/Run (13 each); Shiver (11); Cry (9) & Avoid (Hide); Shake (Shiver); Sob/Weep (cry) \\
Surprise & Jump (15); Gasp (13); Scream (12) & Inhale/Sharp Intake (Gasp); Shout/Yell/Shriek (Scream) \\
\hline
\end{tabular}


Table 2 (on next page)

Main effects and interactions for the mixed factor ANOVA (italics denote significant and marginal results, at $p<0.1$ ).

Main effects and interactions for rating direction $\mathrm{x}$ instruction perspective $\mathrm{x}$ emotion category $x$ typicality, mixed factorial ANOVA (italics denote significant and marginal results, at $p<$ $0.1)$. 
2 Table 2: Main effects and interactions for the rating direction $\times$ instruction perspective $\times$ emotion category $\times$ typicality, mixed

3 factorial ANOVA (italics denote significant and marginal results, at $p<0.1$ ).

\begin{tabular}{|c|c|c|c|c|c|}
\hline Effect & $D F$ & $M S E$ & $F$ & $P$ & $\eta_{p}{ }^{2}$ \\
\hline Category & $(4.34,325.24)$ & 0.53 & 18.93 & $<0.001 *$ & 0.20 \\
\hline Typicality & $(1,75)$ & 1.04 & 696.35 & $<0.001 *$ & 0.90 \\
\hline Instruction Perspective & $(1,75)$ & 2.05 & 6.19 & $<0.015^{*}$ & 0.08 \\
\hline Rating Direction & $(1,75)$ & 2.05 & 5.50 & $<0.022 *$ & 0.07 \\
\hline Category*Instruction Perspective & $(4.34,325.54)$ & 0.53 & 1.42 & 0.23 & 0.02 \\
\hline Category*Rating Direction & $(4.34,325.54)$ & 0.53 & 0.28 & 0.90 & 0.004 \\
\hline Typicality*Instruction Perspective & $(1,75)$ & 1.04 & 0.08 & 0.77 & 0.001 \\
\hline Typicality*Rating Direction & $(1,75)$ & 1.04 & 1.25 & 0.27 & 0.016 \\
\hline Category*Typicality & $(4.25,318.60)$ & 0.45 & 26.79 & $<0.001 *$ & 0.34 \\
\hline Instruction perspective*Rating Direction & $(1,75)$ & 2.05 & 0.37 & 0.55 & 0.005 \\
\hline Category*Instruction Perspective*Rating Direction & $(4.34,325.24)$ & 0.45 & 0.90 & 0.47 & 0.012 \\
\hline Typicality*Instruction Perspectives*Rating Direction & $(1,75)$ & 1.04 & 1.37 & 0.25 & 0.018 \\
\hline Category*Typicality*Instruction Perspective & $(4.25,318.60)$ & 0.45 & 0.90 & 0.47 & 0.0012 \\
\hline Category*Typicality*Rating Direction & $(4.25,318.60)$ & 0.45 & 3.84 & $0.004 *$ & 0.049 \\
\hline Category*Typicality $*$ Instruction Perspective* Rating & $(4.25,318.60)$ & 0.45 & 3.43 & $0.008^{*}$ & 0.044 \\
\hline Direction & & & & & \\
\hline
\end{tabular}




\section{PeerJ Reviewing Manuscript}

5 * Greenhouse-Geisser corrections were applied for 'Category' and 'Category $\times$ Typicality' effects. 


\section{Table 3(on next page)}

Table depicting mean ratings for typical and atypical word pairs

Mean typical and atypical ratings, $t, p$ and $d$ statistics for each emotion category. 
2 Table 3: Mean typical and atypical ratings, $t, p$ and $d$ statistics for each emotion category.

3

\begin{tabular}{lccccc}
\hline $\begin{array}{l}\text { Emotion } \\
\text { Category }\end{array}$ & Typical Mean (SD) & Atypical Mean (SD) & $t$ & $p$ & $d$ \\
\hline Happy & $4.78(0.47)$ & $2.29(0.75)$ & 24.24 & $<0.001$ & 4.0 \\
Surprise & $4.08(1.11)$ & $3.03(0.66)$ & 9.06 & $<0.001$ & 1.58 \\
Sad & $4.47(0.81)$ & $2.19(0.55)$ & 23.86 & $<0.001$ & 3.31 \\
Fear & $4.35(0.80)$ & $3.22(0.70)$ & 13.04 & $<0.001$ & 1.51 \\
Anger & $3.96(1.02)$ & $2.37(0.67)$ & 13.22 & $<0.001$ & 1.85 \\
Disgust & $4.20(1.03)$ & $2.25(0.72)$ & 13.12 & $<0.001$ & 2.21 \\
Totals & $4.31(0.87)$ & $2.56(0.68)$ & - & - & - \\
\hline
\end{tabular}

4

$5 \quad$ * Degrees of Freedom were always $(1,78)$ 
Figure 1 (on next page)

Mean typical and atypical ratings, per emotion category

Mean typical and atypical ratings, per emotion category. Error bars represent $95 \% \mathrm{Cl}$ 
Figure 1: Mean typical and atypical ratings, per emotion label (error bars represent 95\% CI)

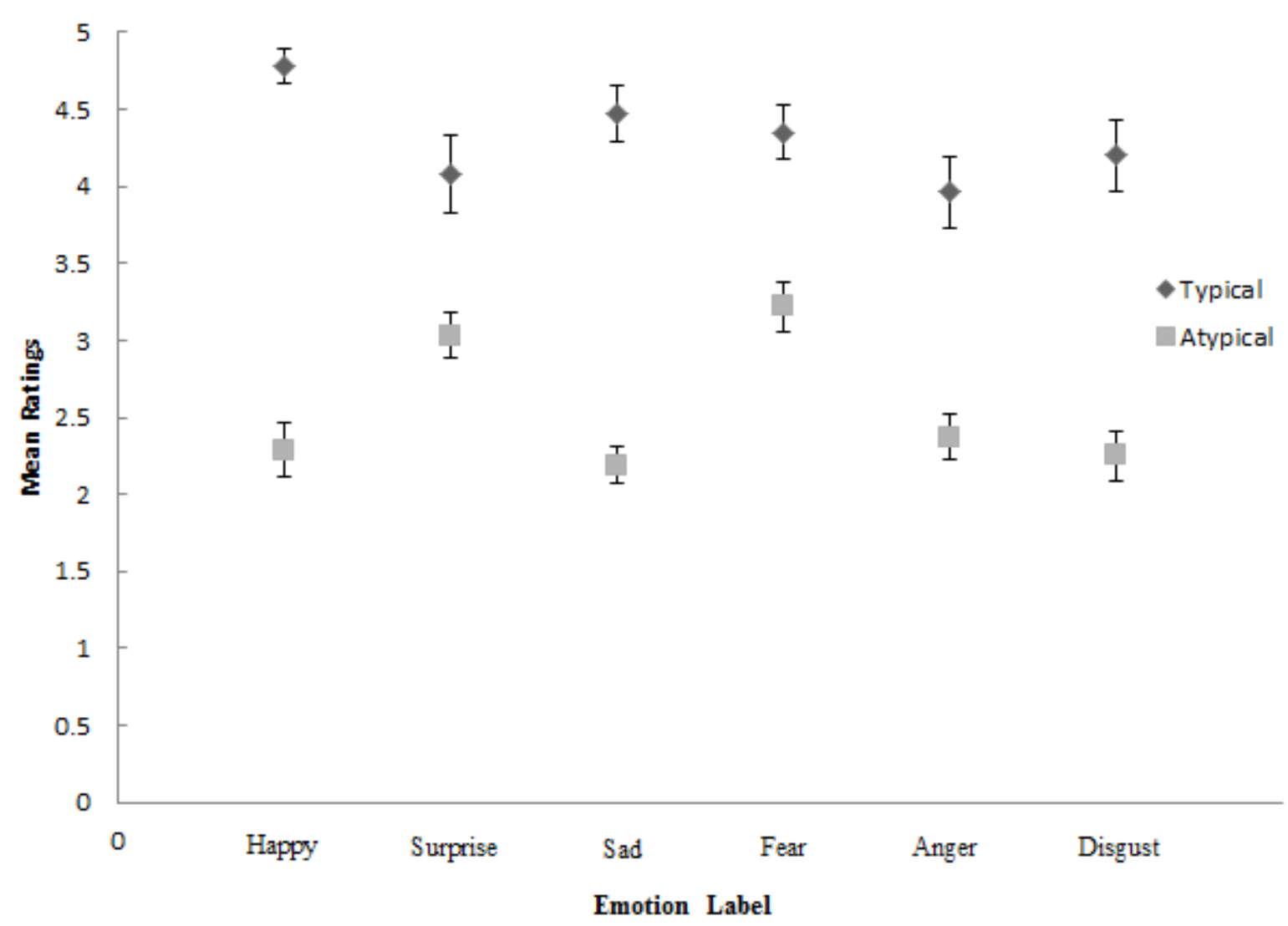


Table 4(on next page)

Mean action word-to-category (A-to-C) and category-to-action word ratings (C-to-A), by typicality and emotion category.

Mean action word-to-category (A-to-C) and category-to-action word ratings (C-to- $A$ ), by typicality and emotion category. $F, p$ and $\eta_{p}^{2}$ statistics are displayed for each effect.

Significant interactions are starred $(p<0.05)$. 
2 Table 4: Mean action word-to-category $(A-t o-C)$ and category-to-action word ratings $\left(C\right.$-to-A), by typicality and emotion category. $F$, $p$ and $\eta_{p}{ }^{2}$ 3 statistics are displayed for each effect. Significant interactions are starred $(p<0.05)$.

4

\begin{tabular}{lllllllll}
\hline Category & \multicolumn{2}{l}{ Mean C-to-A rating (SD) } & \multicolumn{2}{l}{ Mean A-to-C rating (SD) } & $F$ & MSE & $p$ & $\eta_{\mathrm{p}}{ }^{2}$ \\
\cline { 1 - 5 } & \multicolumn{1}{l}{ Typical } & Atypical & Typical & Atypical & & & & \\
\cline { 2 - 5 } Happy & $4.77(0.54)$ & $2.10(0.77)$ & $4.80(0.41)$ & $2.47(0.70)$ & 2.73 & 0.41 & 0.10 & 0.034 \\
Surprise & $3.97(1.18)$ & $2.97(0.79)$ & $4.18(1.03)$ & $3.08(0.51)$ & 0.18 & 0.54 & 0.67 & 0.002 \\
Sad & $4.21(1.00)$ & $2.18(0.59)$ & $4.73(0.45)$ & $2.20(0.51)$ & 7.40 & 0.33 & $0.008^{*}$ & 0.088 \\
Fear & $4.13(0.98)$ & $3.19(0.74)$ & $4.58(0.50)$ & $3.25(0.66)$ & 5.32 & 0.29 & $0.024^{*}$ & 0.065 \\
Anger & $3.90(0.97)$ & $2.17(0.67)$ & $4.03(1.07)$ & $2.58(0.61)$ & 1.37 & 0.57 & 0.25 & 0.017 \\
Disgust & $4.00(1.03)$ & $2.30(0.66)$ & $4.40(1.01)$ & $2.20(0.78)$ & 2.91 & 0.86 & 0.092 & 0.036 \\
Totals & $4.16(0.95)$ & $2.49(0.70)$ & $4.45(0.75)$ & $2.63(0.63)$ & - & - & - & - \\
\hline
\end{tabular}

5

$6 \quad$ * Degrees of freedom were always $(1,77)$. 


\section{Table 5 (on next page)}

Mean third-person and first-person perspective ratings.

Mean third-person and first-person perspective ratings, by rating direction, typicality and emotion category (standard deviations in parenthesis). 
2 Table 5: Mean third-person and first-person perspective ratings, by rating direction, typicality and emotion category (standard 3 deviations in parenthesis).

4

\begin{tabular}{|c|c|c|c|c|c|c|c|c|}
\hline \multirow[t]{3}{*}{ Emotion Category } & \multicolumn{4}{|c|}{ First person ratings (SD) } & \multicolumn{4}{|c|}{ Third person ratings (SD) } \\
\hline & \multicolumn{2}{|c|}{ Category-to-Action Word } & \multicolumn{2}{|c|}{ Action Word-to-Category } & \multicolumn{2}{|c|}{ Category-to-Action Word } & \multicolumn{2}{|c|}{ Action Word-to-Category } \\
\hline & Typical & Atypical & Typical & Atypical & Typical & Atypical & Typical & Atypical \\
\hline Happy & $4.70(0.66)$ & $1.98(0.84)$ & $4.73(0.45)$ & $2.22(0.70)$ & $4.85(0.37)$ & $2.20(0.68)$ & $4.85(0.37)$ & $2.70(0.67)$ \\
\hline Surprise & $3.95(1.23)$ & $2.74(0.95)$ & $4.00(1.17)$ & $2.98(0.61)$ & $4.00(1.12)$ & $3.19(0.51)$ & $4.25(0.97)$ & $3.09(0.42)$ \\
\hline Sad & $4.05(1.31)$ & $1.96(0.60)$ & $4.58(1.03)$ & $2.15(0.56)$ & $4.40(0.52)$ & $2.36(0.54)$ & $4.70(0.47)$ & $2.19(0.50)$ \\
\hline Fear & $3.80(1.20)$ & $3.02(0.89)$ & $4.27(0.72)$ & $2.98(0.79)$ & $4.45(0.51)$ & $3.35(0.48)$ & $4.75(0.44)$ & $3.50(0.38)$ \\
\hline Anger & $3.97(1.18)$ & $2.17(0.80)$ & $3.92(1.09)$ & $2.60(0.75)$ & $4.00(0.73)$ & $2.16(0.53)$ & $4.15(0.93)$ & $2.53(0.47)$ \\
\hline Disgust & $3.58(1.22)$ & $2.39(0.76)$ & $4.42(0.99)$ & $1.90(0.75)$ & $4.40(0.60)$ & $2.21(0.55)$ & $4.35(1.23)$ & $2.45(0.73)$ \\
\hline Totals & $4.01(1.13)$ & $2.38(0.81)$ & $4.32(0.91)$ & $2.47(0.69)$ & $4.35(0.61)$ & $2.58(0.55)$ & $4.51(0.74)$ & $2.74(0.53)$ \\
\hline
\end{tabular}

